\title{
AN UNSTEADY FLOW AND MELTING HEAT TRANSFER OF A NANOFLUID OVER A STRETCHING SHEET EMBEDDED IN A POROUS MEDIUM
}

\author{
K. GANESH KUMAR* \\ Department of Mathematics, SJM Institute of Technology College \\ Chitradurga-577502, Karnataka, INDIA \\ E-mail: ganikganesh@gmail.com \\ B.J. GIREESHA and N.G. RUDRASWAMY \\ Department of Studies and Research in Mathematics, Kuvempu University \\ Shankaraghatta-577 451, Shimoga, Karnataka, INDIA \\ M.R. KRISHNAMURTHY \\ Department of Mathematics, JNN Collage of Engineering \\ Shimoga, Karnataka, INDIA
}

\begin{abstract}
An unsteady flow and melting heat transfer of a nanofluid over a stretching sheet was numerically studied by considering the effect of chemical reaction and thermal radiation. The governing non-linear partial differential equations describing the flow problem are reduced to a system of non-linear ordinary differential equations using the similarity transformations and solved numerically using the Runge-Kutta-Fehlberg fourth-fifth order method. Numerical results for concentration, temperature and velocity profiles are shown graphically and discussed for different physical parameters. Effect of pertinent parameters on momentum, temperature and concentration profiles along with local Sherwood number, local skin-friction coefficient and local Nusselt number are well tabulated and discussed.
\end{abstract}

Key words: melting effect, chemical reaction, nanofluid, porous medium, stretching sheet, unsteady flow.

\section{Introduction}

Flow of an incompressible fluid past a stretching surface is one of the thrust areas of current research in the field of fluid dynamics due to its possible applications in many manufacturing and metallurgical processes of modern industries, such as aerodynamic, extrusion of plastic sheets, continuous stretching of plastic films and artificial fibers, cooling of metallic plate, hot rolling, polymeric extrusion, continuous casting, drawing of continuous filaments through quiescent fluids and annealing and tinning of copper wires, etc. The studies on boundary layer flow past a stretching surface were initiated by Sakiadis [1], who analyzed the problem of boundary layer flow on continuous surfaces which was later developed by Crane [2], by obtaining a closed-form solution for a stretching sheet whose velocity is proportional to the distance from the slit. Furthermore, various flow problems past stretching sheet in the related areas were discussed in [3-9].

It is well known that conventional heat transfer fluids, including oil, water, and ethylene glycol mixture are poor heat transfer fluids. Since the thermal conductivity of these fluids plays an important role on the heat transfer coefficient between the heat transfer medium and the heat transfer surface, an innovative

\footnotetext{
* To whom correspondence should be addressed
} 
technique for improving heat transfer by using ultra-fine solid particles in the fluids has been used extensively during the last several years. Nanofluid, a term introduced by Choi [10], is a base fluid with suspended metallic nano-scale particles called nanoparticles. The characteristic feature of nanofluids is thermal conductivity enhancement, a phenomenon observed by Masuda et al. [11]. This phenomenon suggests the possibility of using nanofluids in advanced nuclear systems. Nanofluids with such relatively high thermal conductivities have attracted enormous interest from researchers due to their potential in enhancement. Buongiorno [12] found that the Brownian diffusion and the thermophoresis effects are the most important and reported conservation laws for nanofluids. In the presence of these two effects Khan and Pop [13] studied the laminar fluid flow problem resulting from the stretching of a flat surface in a nanofluid. An additional information on stretching sheet problem could also be found in the cited works $[14,15]$.

Surprisingly, the problem of coupled heat and mass transfer accompanied by melting effect in porous media has received considerably less attention despite of its importance in the study of geothermal systems, spreading of chemical pollutants generated within the earth's crust, melting of permafrost, creation of ice slurries and the extraction of oil. Especially, in the permafrost research and frozen ground thawing, the melting effect plays an important role. According to the analysis of Walker [16], the phenomenon of permafrost degradation in Arctic Alaska is very critical due to global warming and this result accelerates the greenhouse effect. In two recent studies Epstein and Cho [17] and Kazmierczak et al. [18] gave a theoretical interpretation over forced and natural convection, respectively, on the phenomenon of melting from a flat plate embedded in porous media. Yen and Tien [19], Hayat et al. [20], Gorla et al. [21], Makinde et al. [22] and Kumar et al. [23] have also reported problems of melting process past stretching surfaces by considering aspects on stagnation point flow, viscous dissipation on steady/unsteady stretching surfaces/cylinders. But the problems of melting heat transfer past a stretching surface has been neglected in the region of a porous medium with nanoparticles in action.

However, the problem of an unsteady flow near a stagnation point of a nanofluid with the effect of melting heat transfer and thermal radiation in the region of a porous medium past a stretching surface has remained unsolved. Thus the purpose of this work is to provide a numerical method that can be used to analyze the problems of convective flow of a nanofluid near a stagnation point with heat and mass transport phenomena combined with melting process and thermal radiation in a porous medium and hence its behavior in the boundary layer.

\section{Formulation of the problem}

Consider a steady, incompressible, laminar, two-dimensional boundary layer flow of an incompressible nanofluid past a stretching sheet coinciding with the plane $y=0$ and the flow has been confined to $y>0$. The flow is generated, due to non-linear stretching of the sheet, caused by the simultaneous application of two equal and opposite forces along the $x$ axis. It is assumed that the velocity $U(x, t)$ of the flow external to the boundary layer is proportional to a distance from the stagnation point as shown, i.e., $U(x, t)=\frac{b x}{1-a t}$ and the velocity of the stretching sheet is $U_{w}(x)=\frac{c x}{1-a t}$, where $\mathrm{b}$ is a positive constant and $c$ is a stretching rate. It is also assumed that the temperature of the melting surface be $T_{m}$, while the temperature in the free-stream condition is $T_{1}$ and $T_{1}>T_{m}$ is the nanoparticle volume fraction at the stretching surface and the ambient values of $T$ and $C$ are denoted by $T_{1}$ and $C_{1}$, respectively.

The governing equations for this investigation are based on the balance laws of mass, linear momentum, energy and nanoparticles volume fraction modified to account for the presence of the magnetic field and thermal radiation effects near a stagnation point along with the effect of melting heat transfer in the region of the porous medium. These can be written as 


$$
\begin{aligned}
& \frac{\partial u}{\partial x}+\frac{\partial v}{\partial y}=0 \\
& \frac{\partial u}{\partial t}+u \frac{\partial u}{\partial x}+v \frac{\partial u}{\partial y}=-\frac{1}{p} \frac{d P}{d x}+v \frac{\partial^{2} u}{\partial y^{2}}-\frac{\sigma B_{0}^{2}}{\rho} u+ \\
& +\left(1-c_{\infty}\right) \rho_{f \infty} \beta_{g}\left(T-T_{\infty}\right)+\left(\rho_{p}-\rho_{f \infty}\right) g\left(C-C_{\infty}\right)-\frac{v}{k} u \\
& \frac{\partial T}{\partial t}+u \frac{\partial T}{\partial x}+v \frac{\partial T}{\partial y}=\alpha_{m} \frac{\partial^{2} T}{\partial y^{2}}+\tau\left[D_{B} \frac{\partial C}{\partial y} \frac{\partial T}{\partial y}+\frac{D_{T}}{D_{\infty}}\left(\frac{\partial T}{\partial y}\right)^{2}\right]-\frac{\partial q_{r}}{\partial y} \\
& \frac{\partial C}{\partial t}+u \frac{\partial C}{\partial x}+v \frac{\partial C}{\partial y}=D_{B} \frac{\partial^{2} C}{\partial y^{2}}+\frac{D_{T}}{D_{\infty}} \frac{\partial^{2} T}{\partial y^{2}}-k_{2} C
\end{aligned}
$$

The respective boundary conditions are given by

$$
\begin{aligned}
& u=U_{w}(x, t), \quad T=T_{m}, \quad C=C_{w} \quad \text { at } \quad y=0, \\
& u=0, \quad v=0, \quad T \rightarrow T_{\infty}, \quad C \rightarrow C_{\infty} \quad \text { as } \quad y \rightarrow \infty,
\end{aligned}
$$

and

$$
k\left(\frac{\partial T}{\partial y}\right)_{y=0}=\rho\left[\lambda_{I}+c_{s}\left(T_{m}-T_{0}\right)\right] v(x, 0)
$$

where $\rho$ is the fluid density, $k$ is the porous term, $\lambda_{I}$ is the latent heat of the fluid and $c_{p}$ is the specific heat at constant pressure, $c_{s}$ is the heat capacity of the solid surface. Equation (2.6) states that the heat conducted to the melting surface is equal to the heat of melting plus the sensible heat required to raise the solid temperature $T_{0}$ to its melting temperature.

Employing the generalized Bernoullis equation, in the free stream $U(x, t)=\frac{b x}{1-a t}$, one gets

$$
U \frac{d U}{d x}=-\frac{1}{p} \frac{d P}{d x}-\frac{\sigma B_{0}^{2}}{\rho} u
$$

Using the Rosseland approximation for radiation, radiation heat flux is expressed as

$$
q_{r}=-\frac{4 \sigma^{*}}{3 k^{*}} \frac{\partial T^{4}}{\partial y}
$$

where $\sigma^{*}$ and $k^{*}$ are the Stefan-Boltzman constant and the mean absorption co-efficient, respectively. The temperature differences within the flow are assumed to be sufficiently small so that $T^{4}$ may be expressed as 
a linear function of temperature $T$ using a truncated Taylor series about the free stream temperature $T_{1}$ and by neglecting higher-order terms, we get

$$
T^{4} \approx 4 T T_{\infty}^{3}-T_{\infty}^{4}
$$

The following set of similarity transformations is now introduced to recast the governing partial differential equations into a set of ordinary differential equations

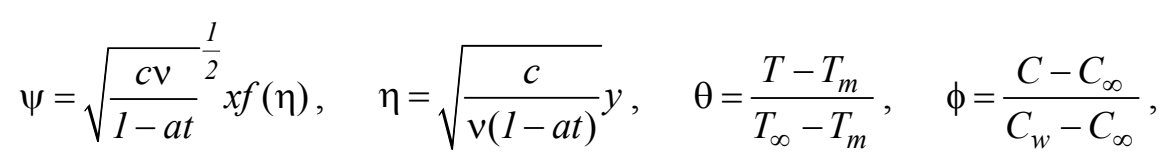

where $\psi$ is the stream function defined in the usual way as $u=\frac{\partial \psi}{\partial y}$ and $v=-\frac{\partial \psi}{\partial x}$ which automatically satisfies the continuity Eq.(2.1). By using this definition, we obtain

$$
u=\frac{c x}{1-a t} f^{\prime}(\eta), \quad v=\sqrt{\frac{c v}{(1-a t)}} f(\eta) .
$$

On substituting Eqs (2.7) to (2.11) in Eqs (2.2) to (2.6) the following nonlinear ordinary differential equations are obtained

$$
\begin{aligned}
& f^{\prime \prime \prime}+f f^{\prime \prime}-\left(f^{\prime}\right)^{2}-A_{0}\left(\frac{\eta}{2} f^{\prime \prime}-f^{\prime}\right)+A^{2}-Q\left(f^{\prime}-A\right)+\operatorname{Ra}_{x}(\theta+N r \phi)+k_{l} f^{\prime}=0, \\
& \frac{1}{\operatorname{Pr}}\left(1+\frac{4}{3} R\right) \theta^{\prime \prime}+f \theta^{\prime}-A_{0} \frac{\eta}{2} \theta^{\prime}+N b \phi^{\prime} \theta^{\prime}+N t\left(\theta^{\prime}\right)^{2}=0, \\
& \phi^{\prime \prime}+\operatorname{Le} f \phi^{\prime}-A_{0} \frac{\eta}{2} \phi+\frac{N t}{N b} \theta^{\prime \prime}-\gamma \phi=0 .
\end{aligned}
$$

The associated boundary conditions are given by

$$
\begin{aligned}
& f^{\prime}(\eta)=1, \quad \operatorname{Pr} f(\eta)+M \theta^{\prime}(\eta)=0, \quad \theta(\eta)=0, \quad \phi(\eta)=0 \quad \text { at } \quad \eta=0, \\
& f^{\prime}(\eta)=A, \quad \theta(\eta)=1, \quad \phi(\eta)=1 \quad \text { as } \quad \eta \rightarrow \infty
\end{aligned}
$$

where a prime denotes the differentiation with respect to $\eta, A=\frac{b}{c}$ is the stretching parameter, $Q=\frac{\sigma B_{0}^{2}}{\rho c}$ is the magnetic parameter, $\operatorname{Ra}_{x}=\frac{\left(1-C_{\infty}\right) \beta_{g}\left(T_{w}-T_{\infty}\right) f_{\infty}}{c^{2} x}$ is the local Rayleigh number, $N r=\frac{\left(\rho_{p}-\rho_{f \infty}\right) g\left(C-C_{\infty}\right)}{\left(1-C_{\infty}\right) \beta_{g}\left(T_{w}-T_{\infty}\right) \rho f_{\infty}}$ is the buoyancy ratio, $k_{l}=\frac{v}{k(1-a t)} b$ is the porosity parameter, $R=\frac{4 \sigma^{*} T_{\infty}^{3}}{k k^{*}}$ 
is the radiation parameter, $\operatorname{Pr}=\frac{v}{\alpha_{m}}$ is the Prandtl number, $N t=\frac{\tau D_{T}\left(T_{w}-T_{\infty}\right)}{v T_{\infty}}$ is the thermophoresis parameter, $N b=\frac{\tau D_{B}\left(C_{w}-C_{\infty}\right)}{v}$ is the Brownian motion parameter, Le $=\frac{v}{D_{B}}$ is the Lewis number, $A_{0}=\frac{a}{c}$ is a parameter that measures the unsteadiness, $\gamma=\frac{k_{2}\left(C_{w}-C_{\infty}\right)}{v}$ is the chemical reaction parameter and $M$ is the dimensionless melting parameter which is defined as

$$
M=\frac{c_{f}\left(T_{\infty}-T_{m}\right)}{\lambda+c_{s\left(T_{m}-T_{0}\right)}} .
$$

Here the melting parameter is a combination of the Stefan numbers $\frac{c_{f}\left(T_{\infty}-T_{m}\right)}{\lambda}$ and $\frac{c_{s}\left(T_{m}-T_{0}\right)}{\lambda}$, of special significance for this type of flow and heat transfer situation are the skin-friction coefficient $\left(c_{f}\right)$ the local Nusselt number $\left(\mathrm{Nu}_{x}\right)$ and the local Sherwood number $\left(\mathrm{Sh}_{x}\right)$.

These physical parameters can be defined in dimensionless form as

$$
c_{f} \sqrt{\mathrm{Re}_{x}}=-f^{\prime \prime}(0), \quad \frac{\mathrm{Nu}_{x}}{\sqrt{\mathrm{Re}_{x}}}=-\left(1+\frac{4}{3} R\right) \theta^{\prime}(0) \quad \text { and } \quad \frac{\mathrm{Sh}_{x}}{\sqrt{\mathrm{Re}_{x}}}=-\phi^{\prime}(0)
$$

where $\operatorname{Re}_{x}=\frac{U_{w} x}{v_{f}}$ signifies the local Reynolds number.

\section{Numerical method}

Equations (2.12) to (2.14) together with the boundary conditions (2.15) form a set of highly nonlinear ordinary differential equations. In order to solve these equation numerically symbolic software Maple has been adopted, which is very efficient in using the well-known RKF-45 order method. In accordance with the boundary layer analysis, the boundary conditions at infinity were replaced by $\eta=6$. Table 1 shows $\mathrm{s}$ comparison of the current solution with the existing solutions. We found a better agreement of the current result with the published work under specific limited cases.

Table 1. Comparison of the numerical solutions for the steady-state flow.

\begin{tabular}{|cc|cc|cc|}
\hline \multirow{2}{*}{$N b$} & $N t$ & \multicolumn{2}{|c|}{ Mustafa et al. $[24]$} & \multicolumn{2}{c|}{ present studies } \\
$\mathrm{Nu}_{x}$ & $\mathrm{Sh}_{x}$ & $\mathrm{Nu}_{x}$ & $\mathrm{Sh}_{x}$ \\
\hline 0.1 & 0.1 & 0.5372 & 0.2244 & 0.53727 & 0.22445 \\
0.5 & & 0.4351 & 0.5275 & 0.43514 & 0.52756 \\
0.5 & 0.5 & 0.3861 & 0.3471 & 0.38615 & 0.34718 \\
& 1 & 0.3343 & 0.1888 & 0.33431 & 0.1889 \\
\hline
\end{tabular}


Table 2. Wall temperature gradient $-\theta^{\prime}(0), f^{\prime \prime}(0)$ and $-\phi^{\prime}(0)$ for the case of assisting flow for different values of the parameters $\operatorname{Pr}$, Le, $N t, N b, N r, M, A_{0}, \lambda, Q, R, k_{l}$ and $\gamma$.

\begin{tabular}{|c|c|c|c|c|c|c|c|c|c|c|c|c|c|c|}
\hline & $\operatorname{Pr}$ & $N b$ & $N t$ & $M$ & $R$ & $Q$ & $\mathrm{Nr}$ & Le & $k_{1}$ & $A_{0}$ & $\gamma$ & $f^{\prime \prime}(0)$ & $-\theta^{\prime}(0)$ & $-\phi^{\prime}(0)$ \\
\hline 0 & 3.2 & 0.5 & 0.5 & 0.5 & 0.5 & 0.1 & 0.45 & 10 & 0.5 & 0.2 & 0.5 & -1.1106 & 1.3737 & 0.0717 \\
\hline 0.1 & & & & & & & & & & & & -1.1019 & 1.3948 & 0.0722 \\
\hline 0.3 & & & & & & & & & & & & -1.0091 & 1.4639 & 0.0716 \\
\hline & 2 & & & & & & & & & & & -1.1099 & 0.8385 & 0.3859 \\
\hline & 2.5 & & & & & & & & & & & -1.1019 & 0.2694 & 1.0616 \\
\hline & 3.2 & & & & & & & & & & & -1.0919 & 1.3948 & 0.0722 \\
\hline & & 0.3 & & & & & & & & & & -1.1068 & 1.22403 & -0.0595 \\
\hline & & 0.5 & & & & & & & & & & -1.0919 & 1.3948 & 0.0722 \\
\hline & & 1 & & & & & & & & & & -1.0621 & 1.7901 & 0.0769 \\
\hline & & & 0.5 & & & & & & & & & -1.1010 & 1.2347 & 0.4275 \\
\hline & & & 0.5 & & & & & & & & & -1.0919 & 1.3948 & 0.0722 \\
\hline & & & 0.8 & & & & & & & & & -1.0783 & 1.6633 & -0.6105 \\
\hline & & & & 0.5 & & & & & & & & -1.0919 & 1.3948 & 0.0722 \\
\hline & & & & 1 & & & & & & & & -1.0608 & 1.0639 & 0.0792 \\
\hline & & & & 1.5 & & & & & & & & -1.0412 & 0.8708 & 0.0348 \\
\hline & & & & & 0.5 & & & & & & & -1.0919 & 1.3948 & 0.0722 \\
\hline & & & & & 1 & & & & & & & -1.1191 & 1.0509 & 0.4109 \\
\hline & & & & & 1.5 & & & & & & & -1.1374 & 0.8428 & 0.6303 \\
\hline & & & & & & 0.1 & & & & & & -1.0919 & 1.3948 & 0.0722 \\
\hline & & & & & & 0.5 & & & & & & -1.2342 & 1.3260 & 0.0708 \\
\hline & & & & & & 1 & & & & & & -1.3922 & 1.2517 & 0.0673 \\
\hline & & & & & & & 0.45 & & & & & -1.0919 & 1.3948 & 0.0722 \\
\hline & & & & & & & 1 & & & & & -1.0576 & 1.4387 & 0.0708 \\
\hline & & & & & & & 2 & & & & & -0.9959 & 1.5055 & 0.0677 \\
\hline & & & & & & & & 10 & & & & -1.0919 & 1.3948 & 0.0722 \\
\hline & & & & & & & & 15 & & & & -1.0815 & 1.4808 & 0.1414 \\
\hline & & & & & & & & 20 & & & & -1.0755 & 1.5322 & 0.1560 \\
\hline & & & & & & & & & 0.5 & & & -1.0919 & 1.3948 & 0.0722 \\
\hline & & & & & & & & & 1 & & & -1.3042 & 1.2755 & 0.0694 \\
\hline & & & & & & & & & 1.5 & & & -1.4896 & 1.1670 & 0.0614 \\
\hline & & & & & & & & & & 0 & & -0.8439 & 1.6719 & 0.0792 \\
\hline & & & & & & & & & & 0.2 & & -0.9474 & 1.4712 & 0.0718 \\
\hline & & & & & & & & & & 0.5 & & -1.1130 & 1.0202 & 0.0324 \\
\hline & & & & & & & & & & & 0 & -0.9318 & 1.5989 & 0.1538 \\
\hline & & & & & & & & & & & 0.5 & -0.9474 & 1.4712 & 0.0718 \\
\hline & & & & & & & & & & & 1 & -0.9598 & 1.3745 & 0.0001 \\
\hline
\end{tabular}

\section{Results and discussion}

Figures 1-26 represent typical numerical results based on the solution of Eqs (2.12) - (2.14). These results are obtained to illustrate the influence of the magnetic field, melting parameter, Brownian motion parameter, thermophoresis parameter, Lewis number, radiation parameter and porosity parameter on velocity, temperature and concentration profiles. 


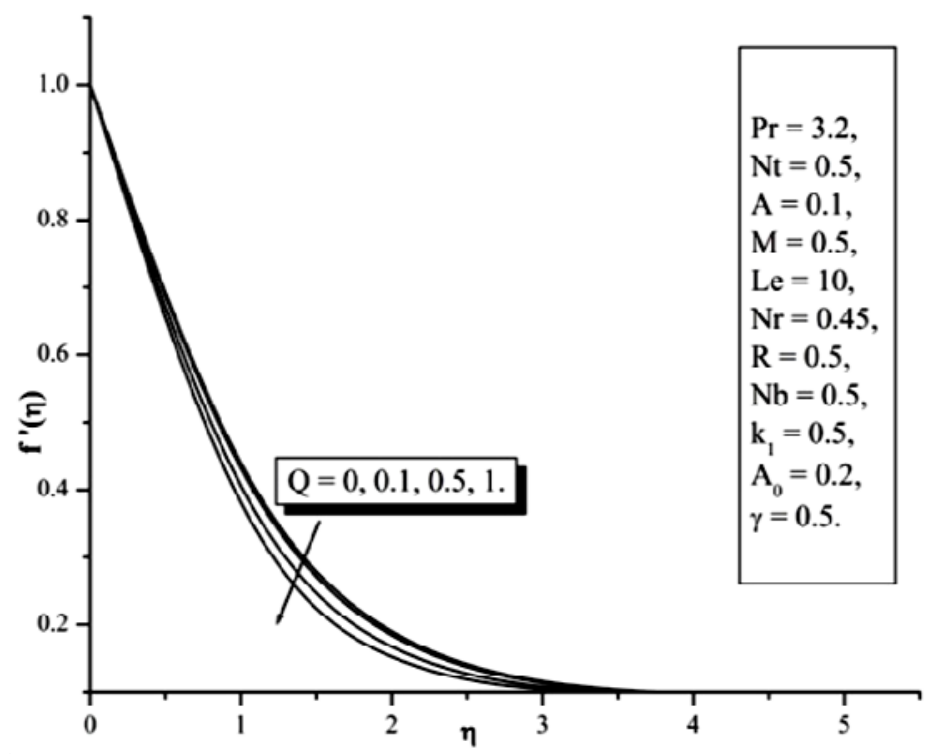

Fig.1. Influence of the magnetic field $(Q)$ on velocity profile.

The velocity profile for different values of the magnetic parameter is shown in the Fig.1. It is observed that an increase in the magnetic field strength decreases the velocity of the nanofluid. This is due to the fact that application of the magnetic field adds a resistive term to the momentum equation which results in the reduction in the velocity of the nanofluid, thereby the momentum boundary layer thickness is decreased.

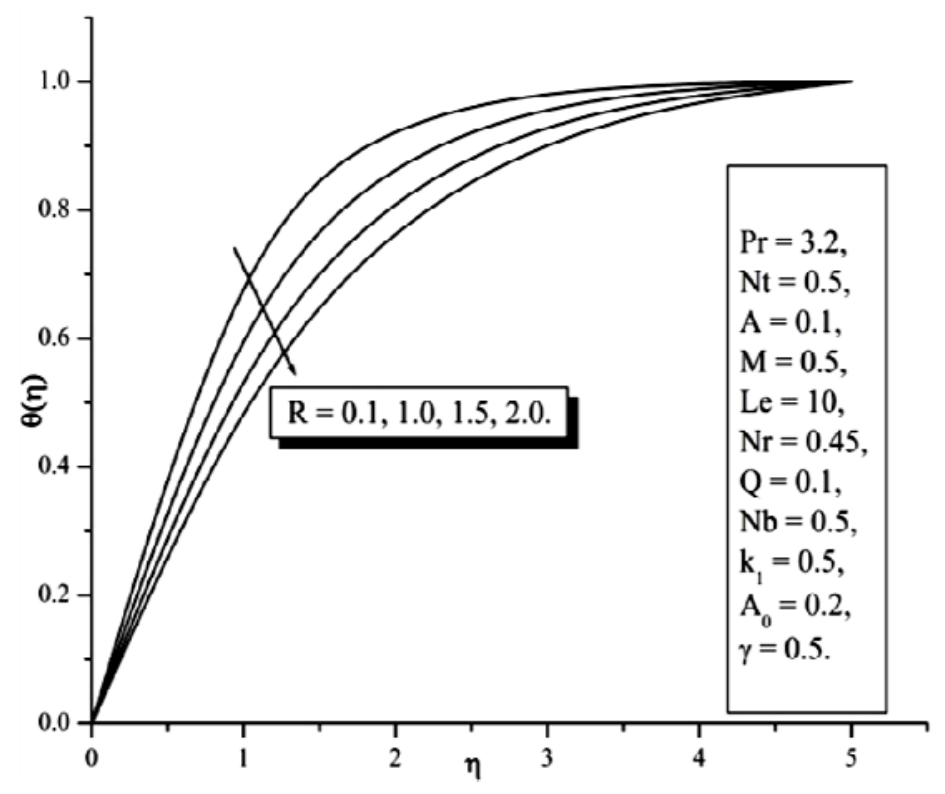

Fig.2. Effect of the thermal radiation parameter on $(R)$ temperature profile.

Figure 2 demonstrates the influence of the thermal radiation parameter on temperature profile. It is found that an increase in $R$ leads to a decrease in the conduction effect and the thermal boundary layer. 


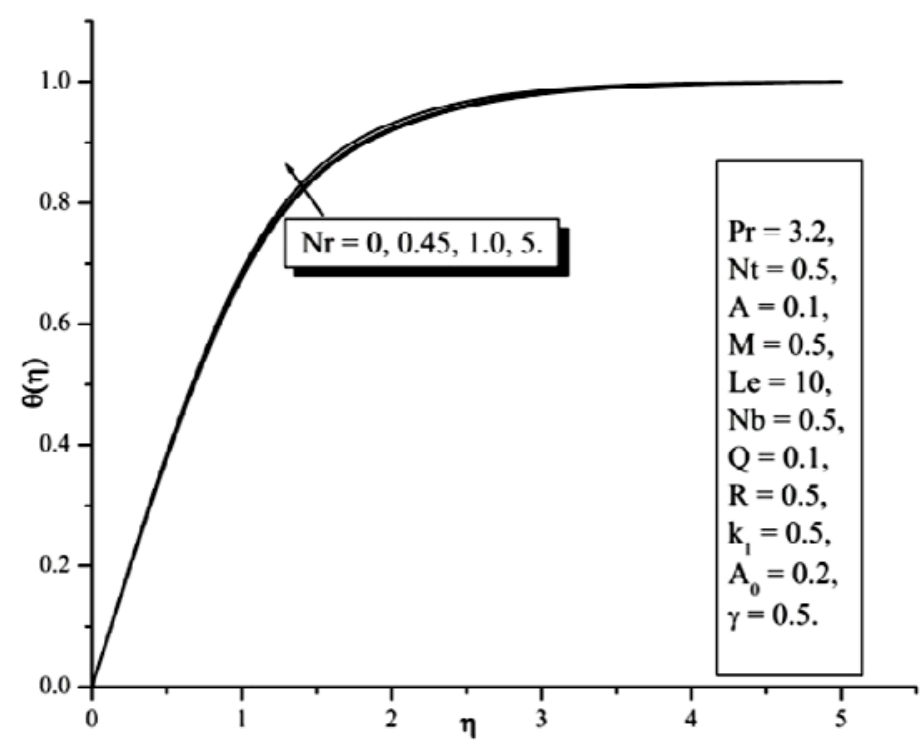

Fig.3. Effect of the bouyancy ratio $(\mathrm{Nr})$ on temperature profile.

Same features could be seen in the boundary region for the increase of the buoyancy ratio $(\mathrm{Nr})$ parameter. It is observed that as the buoyancy ratio increases temperature of the nanofluid in the boundary influences in building thermal boundary layer of the nanofluid is as shown in Fig.3.

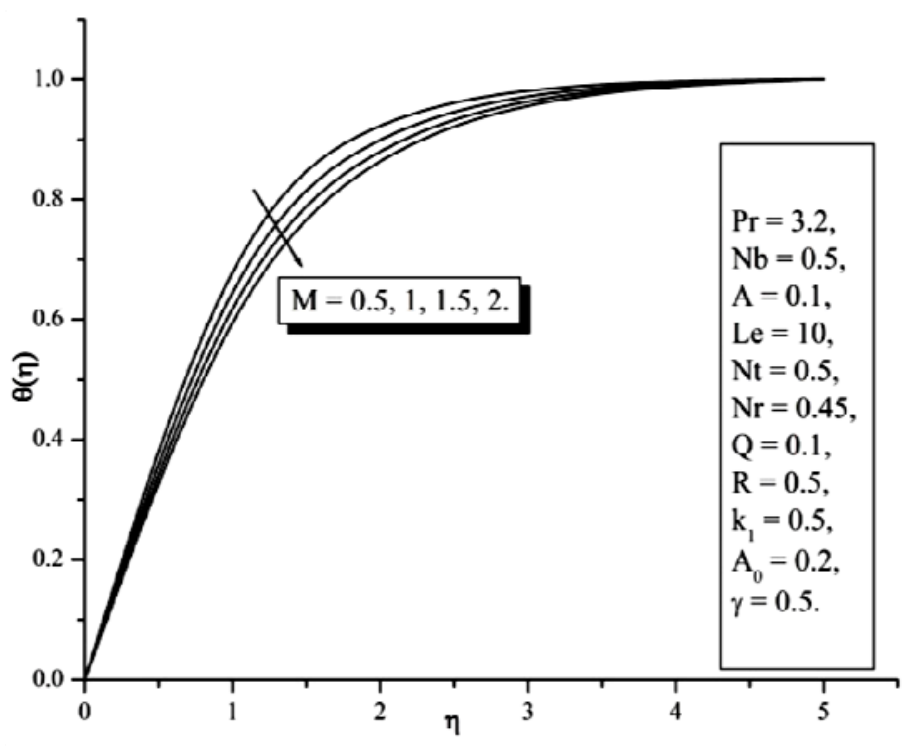

Fig.4. Effect of the melting parameter $(M)$ on temperature profile.

Figure 4 reveals the temperature distribution for different values of the melting parameter $(M)$. It is observed that increase in the values of melting parameter causes a decrease in the temperature distribution. This behavior means that, a small melting parameter disturbance in the free stream temperature $T_{\infty}$ approximately equal to the surface temperature $T_{m}$ i.e., $T_{\infty} \approx T_{m}$ which implies an enhancement in the temperature distribution. 


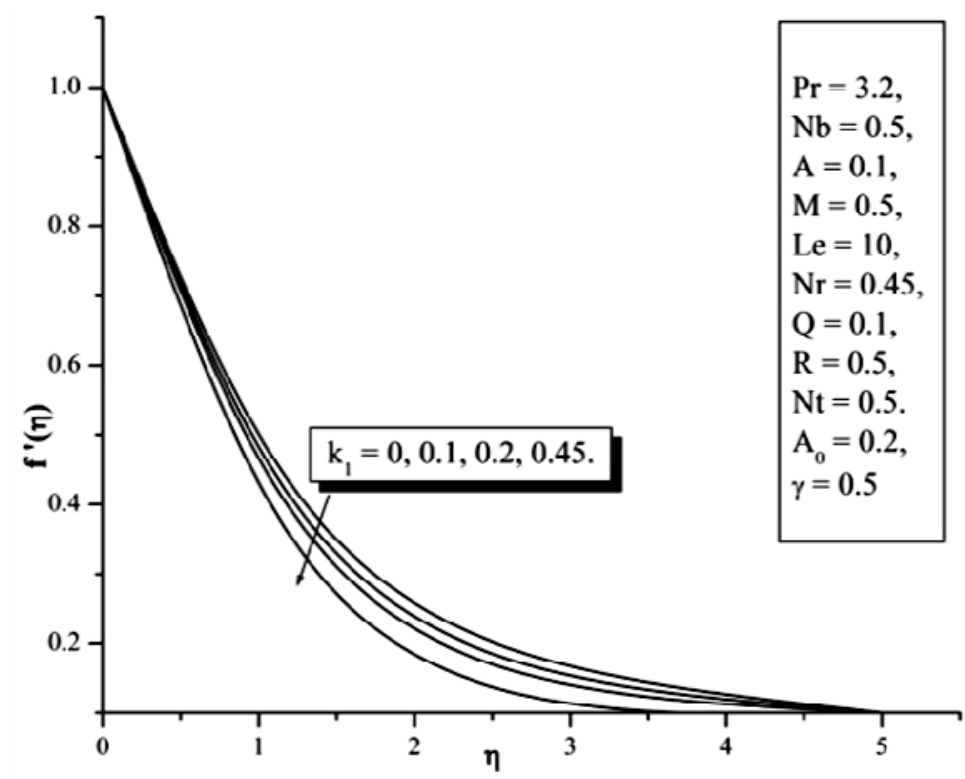

Fig.5. Effect of the porosity parameter $\left(k_{l}\right)$ on velocity profile.

Figure 5 shows the influence of the porosity parameter on velocity profile. The momentum boundary layer is affected by the porosity parameter. It is seen that the thickness of the momentum boundary layer decreases with an increase in the porosity parameter. This is due to the presence of porous medium which increases the resistance to the flow causing a decrease in the fluid velocity. As a result, velocity in the boundary layer decreases.

From the definition of Prandtl number it is quite obvious that, a large Pr has a lower thermal diffusivity. Due to the effect of the melting parameter, the thermal boundary layer thickness increases by an increasing values of $\mathrm{Pr}$ and also enhances the thermal boundary layer thickness as shown in Fig.6.

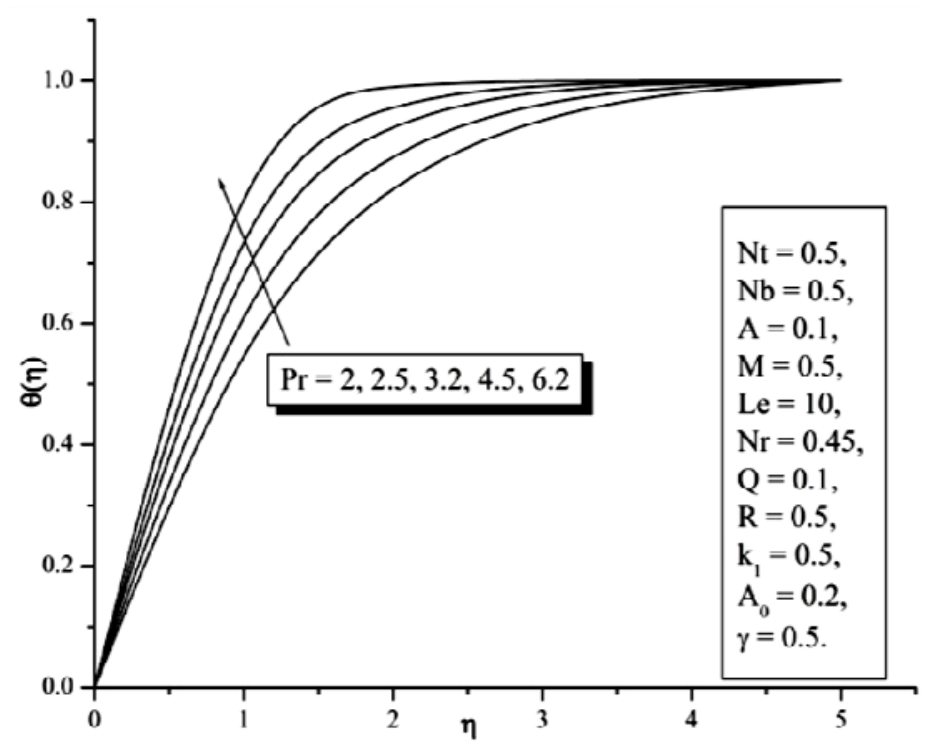

Fig.6. Effect of the Prandtl number (Pr) on temperature profile. 


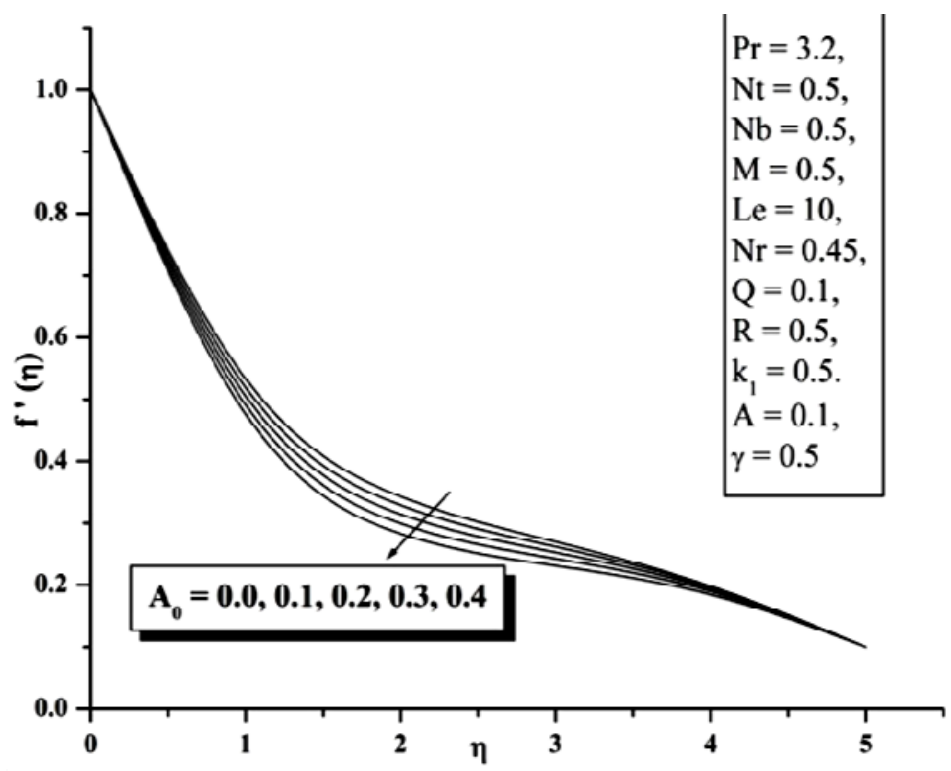

Fig.7. Effect of the unsteady parameter $\left(A_{0}\right)$ on velocity profile.

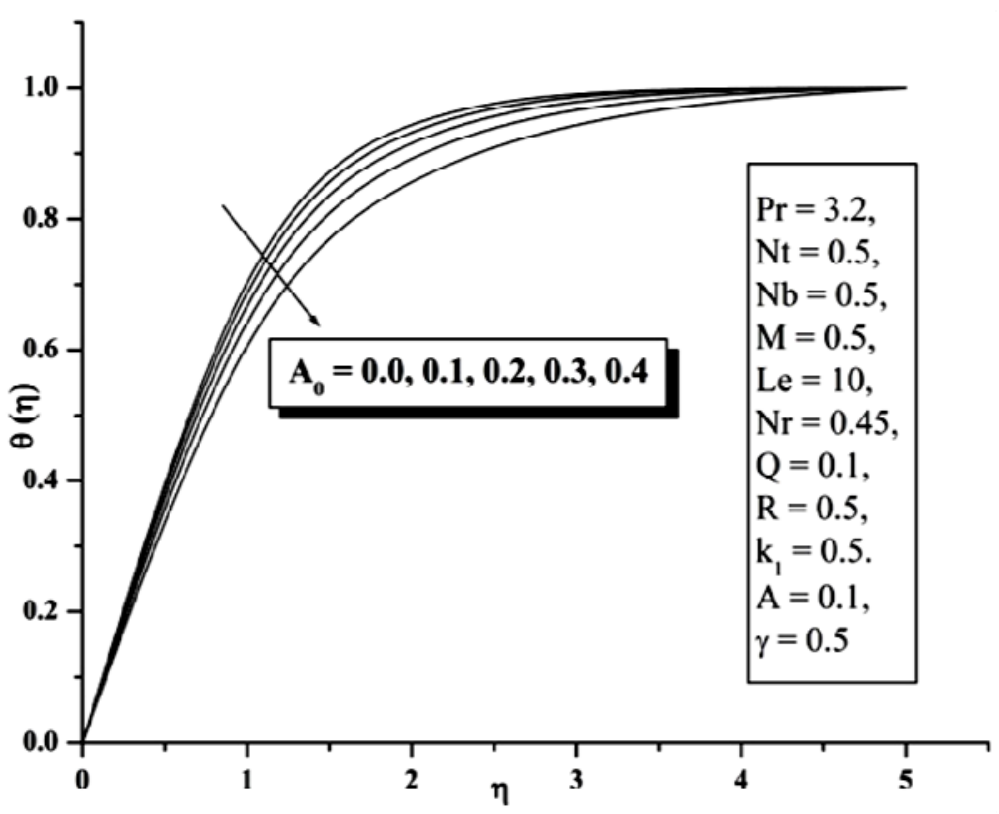

Fig.8. Effect of the unsteady parameter $\left(A_{0}\right)$ on temperature profile.

Influence of the unsteady parameter on velocity and temperature profiles are shown in Figs 7 and 8. It is clear that, an increase in the unsteady parameter decreases the momentum and thermal boundary layer thicknesses resulting in a reduction of both the velocity and temperature. This shows an important fact that the rate of cooling is much faster for higher values of the unsteady parameter whereas, it may take longer time in steady flows. 


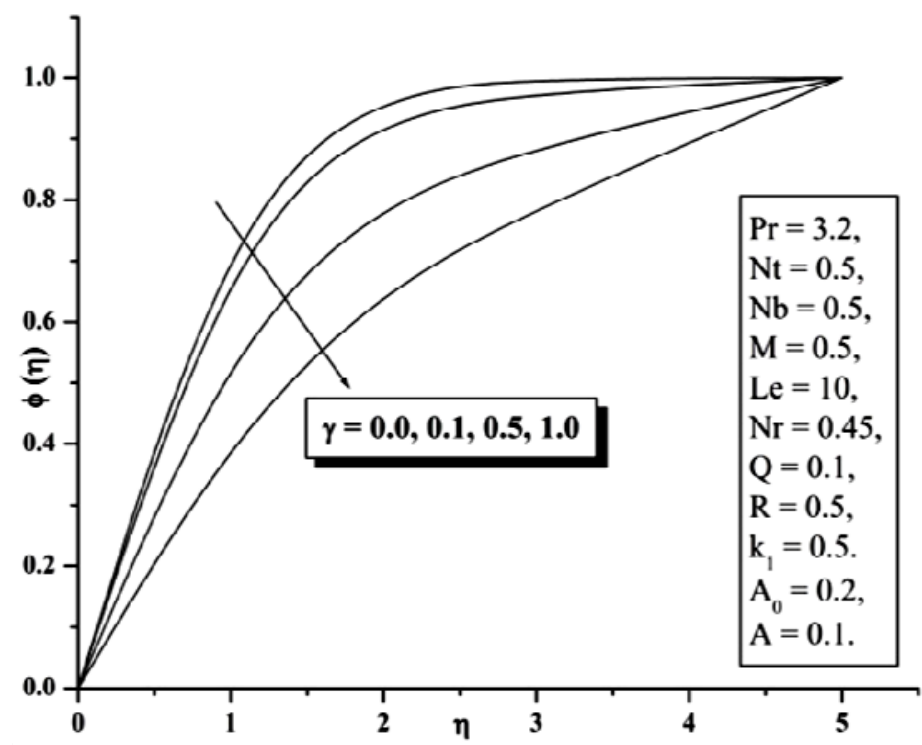

Fig.9. Effect of chemical reaction $\gamma$ on concentration profile.

Figure 9 shows the effect of chemical reaction on the nanoparticle concentration profile. It is seen that the nanoparticle volume fraction of the fluid decreases with an increase in chemical reaction parameter. In particular, the nanoparticle volume fraction of the fluid gradually changes from a higher value to a lower value only when the strength of the chemical reaction is higher than the thermophoresis particle deposition $N t$. For nanoparticle volume characteristics mechanism, an interesting result is seen with the large distortion of the nanoparticle volume field. All these physical behaviors are due to the combined effects of the strength of the Brownian motion and thermophoresis particle deposition.

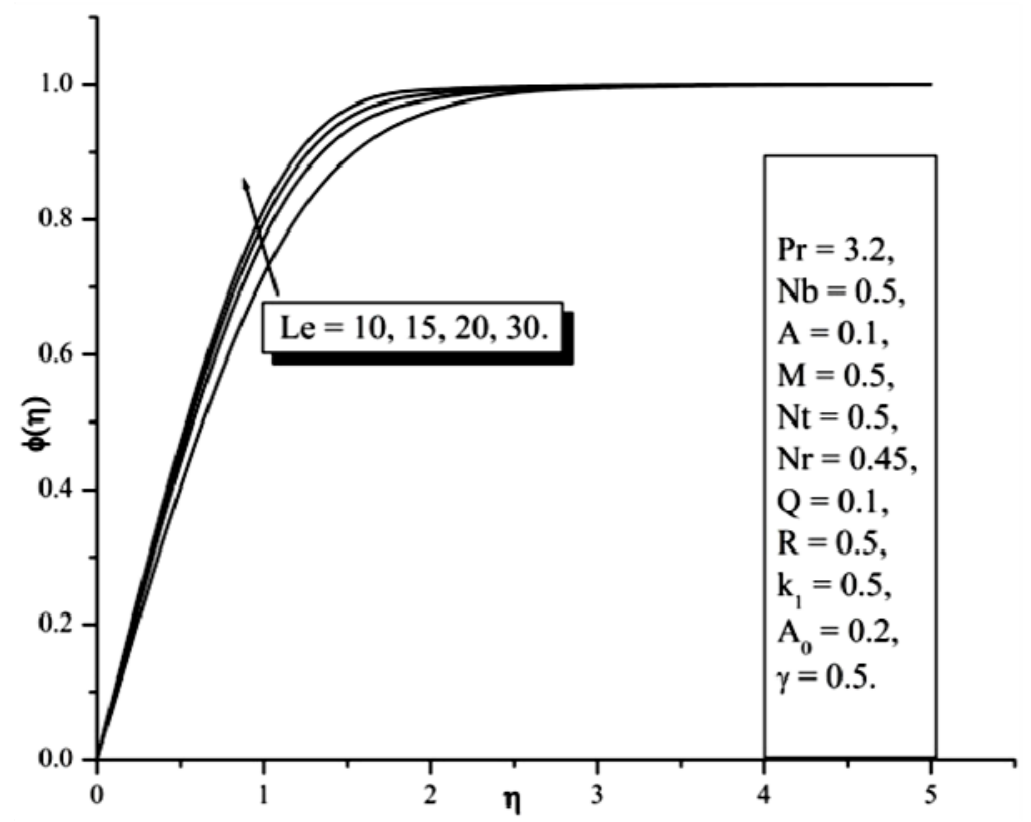

Fig.10. Effect of the Lewis number (Le) on concentration profile. 
It is noticed from Fig. 10 that the presence of the Lewis number increases the concentration profile corresponding to an increase in boundary layer thickness. This is probably due to the fact that mass transfer rate decreases as the Lewis number increases.

\section{Conclusion}

The problem of stagnation point flow of a nanofluid with the effect of melting heat transfer and thermal radiation in a porous medium has been studied with the help of the Runge-Kutta-Fehlberg-45 method and the outcomes of the result are listed below:

- the thermal boundary layer thickness and the temperature is found to decrease as the melting parameter increases;

- it is found that the temperature profile is an increasing function in view of Le;

- velocity of the nanofluid at the boundary decreases for the increase in the porosity parameter;

- increase in the value of the unsteady parameter decreases both the temperature and velocity of the fluid in the boundary;

- the concentration boundary layer is significantly suppressed by $\gamma$.

\section{Nomenclature}

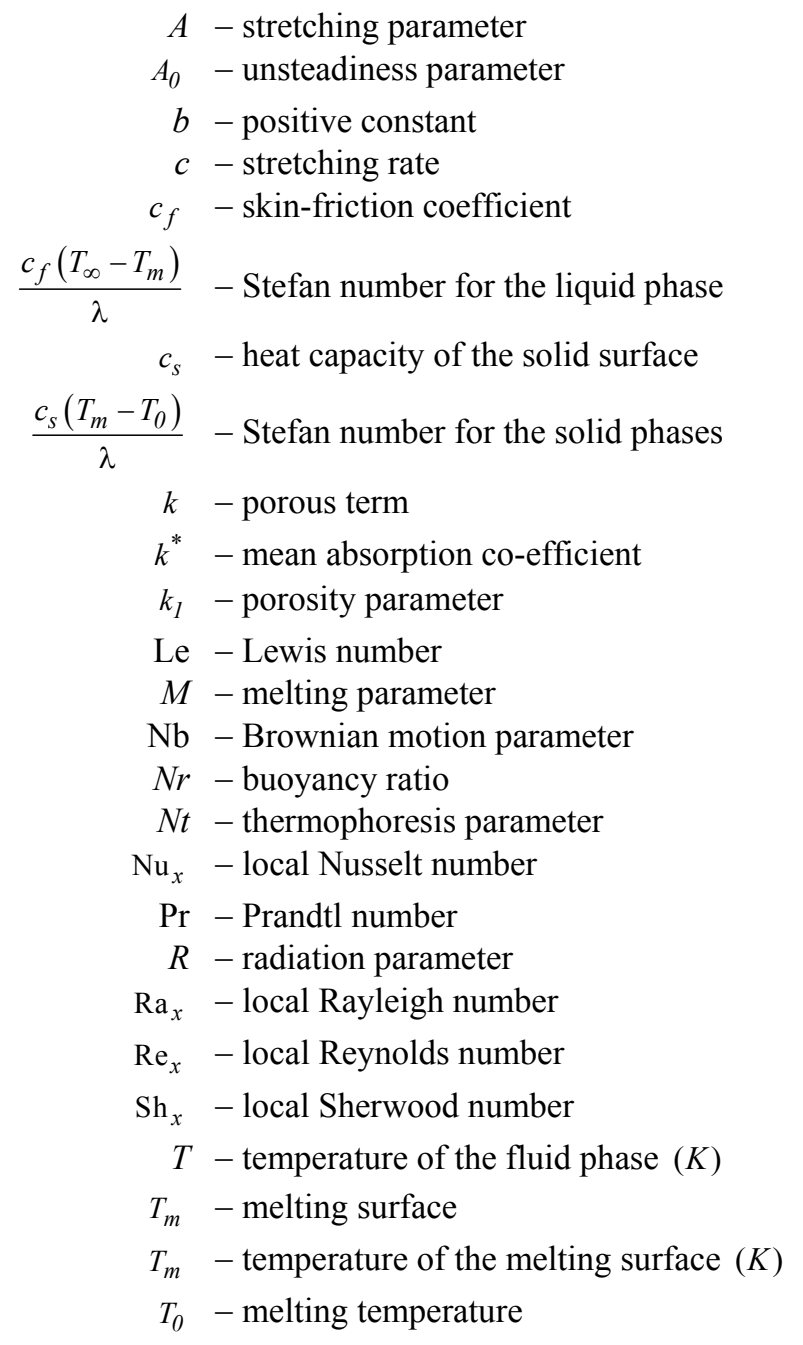


$T_{1}$ - temperature in the free-stream condition

$T_{\infty} \quad$ - ambient fluid temperature $(K)$

$u, v \quad$ - velocity component

$Q$ - magnetic parameter

$q_{r} \quad$ - radiation heat flu

$x$ - coordinate along the plate $(m)$

$y$ - coordinate normal to the plate $(\mathrm{m})$

$\gamma$ - chemical reaction parameter

$\lambda_{1}-$ latent heat of the fluid

$\rho$ - fluid density

$\sigma^{*}-$ Stefan-Boltzman constant

$\psi$ - stream function

\section{References}

[1] Sakiadis B.C. (1961): Boundary layer behavior on continuous solid surfaces: I Boundary layer equations for two dimensional and axisymmetric flow, II. Boundary layer on a continuous flat surface. - AICHE J., vol.7, pp.221225.

[2] Crane L.J. (1970): Flow past a stretching plate. - ZAMP, vol.21, pp.645-647.

[3] Gupta P.S. and Gupta A.S. (1977): Heat and mass transfer on a stretching sheet with suction or blowing. - Can. J. Chem. Eng., vol.55, pp.744-746.

[4] Kumar K.G., Gireesha B.J., Manjunatha S. and Rudraswamy N.G. (2017): Effect of nonlinear thermal radiation on double-diffusive mixed convection boundary layer flow of viscoelastic nanofluid over a stretching sheet. - Int. J. of Mech. and Mat. Eng., vol.12, No.1, pp.18.

[5] Kumar K.G., Gireesha B.J., Rudraswamy N.G. and Manjunatha S. (2017): Radiative heat transfers of Carreau fluid flow over a stretching sheet with fluid particle suspension and temperature jump. - Results in Physics, vol.7, pp.3976-3983.

[6] Kumar K.G., Gireesha B.J. and Gorla R.S.R. (2018): Flow and heat transfer of dusty hyperbolic tangent fluid over a stretching sheet in the presence of thermal radiation and magnetic field. - Int. J. of Mech. and Mat. Eng., vol.13, No.1, pp.1-11.

[7] Kumar K.G., Gireesha B.J., Ramesh G.K. and Rudraswamy N.G. (2018): Double-diffusive free convective flow of Maxwell nanofluid past a stretching sheet with nonlinear thermal radiation. - Journal of Nanofluids, vol.7, No.3, pp.499-508.

[8] Krishnamurthy M.R., Kumar K.G., Gireesha B.J. and Rudraswamy N.G. (2018): MHD flow and heat transfer (PST and PHF) of dusty fluid suspended with alumina nanoparticles over a stretching sheet embedded in a porous medium under the influence of thermal radiation. - Journal of Nanofluids, vol.7, No.3, pp.527-535.

[9] Kumar K.G., Rudraswamy N.G., Gireesha B.J. and Manjunatha S. (2017): Non-linear thermal radiation effect on Williamson fluid with particle-liquid suspension past a stretching surface. - Results in Physics, vol.7, pp.31963202.

[10] Choi S.U.S. (1995): Enhancing thermal conductivity of fluids with nanoparticle. - In: D.A. Siginer, H.P. Wang (Eds.), Developments and Applications of Non-Newtonian Flows, The ASME New York, FED, vol.231/MD vol.66, pp.99-105.

[11] Masuda H., Ebata A., Teramae K. and Hishinuma N. (1993): Alteration of thermal conductivity and viscosity of liquid by dispersing ultra-fine particles. - T NetsuBussei, vol.7, pp.227-233.

[12] Buongiorno J. (2005): Convective transport in nanofluids. - ASME J. Heat Transfer, vol.128, pp.240-250. 
[13] Khan W.A. and Pop I. (2010): Boundary-layer flow of a nanofluid past a stretching sheet. - Int. J. Heat Mass Transfer, vol.53, pp.2477-2483.

[14] Kumar K.G., Ramesh G.K., Gireesha B.J. and Gorla R.S.R. (2017): Characteristics of Joule heating and viscous dissipation on three-dimensional flow of Oldroyd B nanofluid with thermal radiation. - Alexandria Engineering Journal, vol.57, No.3, pp.2139-2149. https://doi.org/10.1016/j.aej.2017.06.006.

[15] Kumar K.G., Gireesha B.J., Krishnamurthy M.R. and Prasannakumara B.C. (2018): Impact of convective condition on Marangoni convection flow and heat transfer in Casson nanofluid with uniform heat source sink. Journal of Nanofluids, vol.7, No.1, pp.108-114.

[16] Walker G. (2007): A world melting from the top down. - Nature, vol.446, pp.718-721.

[17] Epstein M. and Cho D.H. (1976): Laminar film condensation on a vertical melting surface. - ASME J. Heat Transfer, vol.98, pp.108-113.

[18] Kazmierczak M., Poulikakos D. and Pop I. (1986): Melting from a flat plate embedded in a porous medium in the presence of steady natural convection. - Numerical Heat Transfer, vol.10, pp.571-581.

[19] Yen Y.C. and Tien C. (1963): Laminar heat transfer over a melting plate, the modified Leveque problem. - J. Geophys. Res., vol.68, pp.3673-3678.

[20] Hayat T., Iqbal Z., Mustafa M. and Hendi A.A. (2013): Melting heat transfer in the stagnation-point flow of third grade fluid past a stretching sheet with viscous dissipation. - Thermal Science, vol.17, No.3, pp.865-875.

[21] Gorla R.S.R., Chamkha A.J. and Meshaiei E.A. (2012): Melting heat transfer in a nanofluid boundary layer on a stretching circular cylinder. - J. Naval Architecture Marine Engg., vol.9, pp.1-10.

[22] Makinde O.D., Kumar K.G., Manjunatha S. and Gireesha B.J. (2017): Effect of nonlinear thermal radiation on MHD boundary layer flow and melting heat transfer of micro-polar fluid over a stretching surface with fluid particles suspension. - Defect and Diffusion Forum, vol.378, pp.125-136.

[23] Kumar K.G., Gireesha B.J., Gorla R.S.R. and Rudraswamy N.G. (2017): Melting heat transfer of hyperbolic tangent fluid over a stretching sheet with fluid particle suspension and thermal radiation. - Communications in Numerical Analysis, vol.2017, No.2, pp.125-140.

[24] Mustafa M., Hayat T. and Alsaedi A. (2013): Unsteady boundary layer flow of nanofluid past an impulsively stretching sheet. - J. Mechanics, vol.29, No.3, pp.423-432.

Received: October 18, 2017

Revised: September 5, 2018 